\title{
Widening the Lens
}

\author{
Introduction
}

Agomoni Ganguli-Mitra

The sheer diversity of topics in health research makes for a daunting task in the development, establishment, and application of oversight mechanisms and various methods of governance. The authors of this section illustrate how this task is made even more complex by emerging technologies, applications and context, as well as the presence of a variety of actors both in the research and the governance landscape. Nevertheless, key themes emerge, and these sometimes trouble existing paradigms and parameters, and shift and widen our regulatory lenses. A key anchor is the relationship between governance and time: be it the urgent nature of research conducted in global health emergencies; the appropriate weight given to historical data in establishing evidence, anticipating future risk, benefit or harm; or the historical and current forces that have shaped regulatory structures as we meet them today. The perspectives explored in this section can be seen to illustrate different kinds of liminality, which result in regulatory complexity but also offer potential for new kinds of imaginaries, norms and processes.

A first kind of shift in lens is created by the nature of research contexts: for example, whether research is carried out in labs, in clinical settings, traditional healing encounters or, indeed, in a pandemic. These spaces might be the site where values, interests or rules conflict, or they might be characterised by the absence of regulation. Additional tension might be brought about in the interaction of what is being regulated, with how it is being regulated: emerging interventions in already established processes, traditional interventions in more recently developed but strongly established paradigms, or marginal interventions precipitated to the centre by outside forces (crises, economic profit, unexpected findings, imminent or certain injury or death). These shifts give rise to considerations of flexibility and resilience in regulation, of the legitimacy and authority of different actors, and the epistemic soundness in the development and deployment of innovative, experimental, or less established practices.

In Chapter 28, Ho addresses the key concept of risk, and its role within the governance of artificial intelligence (AI) and machine learning (ML) as medical devices. Using the illustration of AI/ML as clinical decision support in the diagnosis of diabetic retinopathy, the author situates their position in qualified opposition to those who perceive governance as an impediment to development and economic gain and those who favour more oversight of AI/ML. In managing such algorithms as risk objects in governance, Ho advocates a governance structure that recharacterises risk as a form of iterative learning process, rather than a rule-based one-time evaluation and regulatory approval based on the quantification of future risk.

The theme of regulation as obstacle is also explored in the following chapter (Chapter 29) by Lipworth et al., in the context of autologous mesenchymal stem cell-based interventions. Here, 
too, the perspective of the authors is set against those who see traditional governance and translational pathways as an impediment to addressing life-threatening and debilitating illnesses. They also resist the reimagination of healthcare as a marketplace (complete with aggressive marketing and dubious claims) where the patient is seen as a consumer, and the decision to access emerging and novel (unproven and potentially risky) interventions merely as a matter of shared decision-making between patient and clinician. The authors recommend the strengthening a multipronged governance framework, which includes professional regulation, marketplace regulation, regulation of therapeutic products, and research oversight.

In Chapter 30, Haas and Cloatre also explore the difficult task of aligning interventions and products within established regulatory and translational pathways. Here, however, the challenge is not novel or emerging interventions, but traditional or non-conventional medicine, which challenges establishes governance frameworks based on the biomedical paradigm, and yet which millions of patients worldwide rely on as their primary form of healthcare. Here, uncertainty relates to the epistemic legitimacy of non-conventional forms of knowledge gathering. Actors in conflict with established epistemic processes are informed by historical and contextual evidence and practices that far predate the establishment of current frameworks. Traditional and nonconventional interventions are, nevertheless, pushed towards hegemonic governance pathways, often in the 'scientised and commercial' forms, in order to gain recognition and legitimacy.

When considering pathways to legitimacy, a key role is played by ethics, in its multiple forms. In Chapter 31, Pickersgill explores ethics in its multiple forms through the eyes of neuroscience researchers, who in their daily practice experience the ethical dimensions of neuroscience and negotiate ethics as a regulatory tool. Ethics can be seen as obstacle to good science, and the (institutional) ethics of human research is often seen as prone to obfuscation and in lack of clear guidance. This results in novel practices and norms within the community, which are informed by a commitment to doing the right thing and by institutional requirements. In order to minimise potential subversion (even well-meant) of ethics in research, Pickersgill advocates the development of governance that arises not only from collaborations between scientists and regulators but also those who can act as critical friends to both of these groups of actors.

Ethics guidance and ethical practices are also explored by Ganguli-Mitra and Hunt (Chapter 32), this time in the context of research carried out in global health emergencies (GHEs). These contexts are characterised by various factors that complicate ethical norms and practices, as well as trouble existing frameworks and paradigms. GHEs are sites of multiple kinds of practices (humanitarian, medical, public health, development) and of multiple actors, whose goals and norms of conduct might be in conflict in a context that is characterised by urgency and high risk of injury and death. Using the examples of recent emergencies, the authors explore the changing nature of ethics and ethical practices in extraordinary circumstances.

In the final chapter of this section (Chapter 33), Arzuaga offers an illustration of regulatory development, touching upon the many actors, values, interests, and forces explored in the earlier chapters. Arzuaga reports on the governance of advanced therapeutic medicinal products (ATMPs) in Argentina, moving from a situation of non-intervention on the part of the state, to the establishment of a governance framework. Here, the role of hard and soft law as adding both resilience and flexibility to regulation is explored, fostering innovation without abdicating ethical concerns. Arzuaga describes early, unsuccessful attempts at regulating stem cell-based interventions, echoing the concerns presented by Lipworth et al., before exploring a more promising exercise in legal foresighting, which included a variety of actors and collaboration, as well a combination of top-down models and bottom-up, iterative processes. 